\title{
Distinct Functions of acj6 Splice Forms in Odor Receptor Gene Choice
}

\author{
Lei Bai and John R. Carlson \\ Department of Molecular, Cellular, and Developmental Biology, Yale University, New Haven, Connecticut 06520
}

Individual olfactory receptor neurons (ORNs) selectively express one or a small number of odor receptors from among a large receptor repertoire. The expression of an odor receptor dictates the odor response spectrum of the ORN. The process of receptor gene choice relies in part on a combinatorial code of transcription factors. In Drosophila, the POU domain transcription factor Acj6 is one element of the transcription factor code. In acj6 null mutants, many ORNs do not express an appropriate odor receptor gene and thus are not correctly specified. We find that acj6 is alternatively spliced to yield many structurally distinct transcripts in the olfactory organs. We generate flies that express single splice forms of acj6 in an $a c j 6^{-}$background. We find that different splice forms are functionally distinct; they differ in their abilities to specify ORN identities. Some individual splice forms can fully rescue the specification of some ORNs. Individual splice forms can function both positively and negatively in receptor gene regulation. ORNs differ in their requirements for splice forms; some are not fully rescued by any single splice form tested, suggesting that some ORNs may require the combinatorial action of multiple splice forms. Late expression of some acj6 splice forms is sufficient to rescue some ORN classes, consistent with a direct role for Acj6 isoforms in receptor gene expression. The results indicate that alternative splicing may add another level of richness to the regulatory code that underlies the process of odor receptor gene choice.

\section{Introduction}

A remarkable problem in molecular neurobiology is how an olfactory receptor neuron (ORN) activates one of a large family of odor receptor genes while simultaneously repressing all the others. The selection of a receptor gene dictates the odor specificity of the ORN, and the orchestration of this process across the entire population of ORNs underlies the sense of smell.

The problem of receptor gene choice is faced by animals ranging from insects to mammals (Fuss and Ray, 2009). The Drosophila genome contains 60 Or (Odorant receptor) genes, and each ORN expresses one or a small number of them. Most Or genes are expressed in one of the two adult olfactory organs, the antenna and the maxillary palp; the larval olfactory organ expresses a subset of Or genes that partially overlaps with the adult repertoire (Couto et al., 2005; Fishilevich et al., 2005; Kreher et al., 2005). In the adult, ORNs of different functional classes are compartmentalized into olfactory sensilla in stereotyped combinations (de Bruyne et al., 1999, 2001). The maxillary palp is populated by three types of sensilla, $\mathrm{pb} 1, \mathrm{pb} 2$, and $\mathrm{pb} 3$, each of which contains ORNs of two classes (pb1A and pb1B, pb2A and pb2B, pb3A and $\mathrm{pb} 3 \mathrm{~B}$, respectively). These six ORN classes each express a single Or gene, except that pb2A coexpresses two Or genes (Goldman et al., 2005).

\footnotetext{
Received Dec. 20, 2009; revised Feb. 8, 2010; accepted Feb. 24, 2010.

This work was supported by grants from the National Institutes of Health and a McKnight Investigator Award (J.R.C.). We thank Sarah Certel for UAS-acj6-F and UAS-acj6-M transgenic flies, Scott Kreher for discussion, and Tong-wey Koh for critical reading of this manuscript.

Correspondence should be addressed to Dr. John R. Carlson, Department of Molecular, Cellular, and Developmental Biology, Yale University, P.O. Box 208103, New Haven, CT 06520-8103. E-mail: john.carlson@yale.edu.

DOI:10.1523/JNEUROSCI.6292-09.2010

Copyright $\odot 2010$ the authors $\quad 0270-6474 / 10 / 305028-09 \$ 15.00 / 0$
}

Odor receptor gene choice in Drosophila depends on the combinatorial action of transcription factors, which read a combinatorial code of regulatory elements flanking the receptor genes (Ray et al., 2007, 2008; Tichy et al., 2008; Bai et al., 2009). The transcription factor that has been characterized in most detail is Acj6, a conserved POU-domain transcription factor (Clyne et al., 1999b) that regulates Or genes both positively and negatively (Bai et al., 2009). In acj6 mutants, a large subset of ORNs lose their odor specificities as a result of the loss of receptor expression; some neurons acquire a novel response profile that results from ectopic receptor expression. Acj6 selectively binds to a site in the promoter regions of Or genes that it regulates.

acj6 undergoes alternative splicing (Certel et al., 2000), raising the possibility that different splice forms might have different functions in the process of receptor gene choice. We identify 13 alternative transcripts of acj6, of which eight are expressed in the maxillary palp. We generate flies that express single splice forms of acj6 in an acj6 background. We find that splice forms are functionally distinct; they differ in their ability to specify neuronal identities. Some individual splice forms are able to rescue fully the odor response properties of some ORNs. Individual splice forms can regulate Or genes both positively and negatively. The expression of some splice forms leads to ectopic expression of larval odor receptors. Different ORNs differ in their requirements for acj6 splice forms. Our results support a model in which alternative splicing adds another degree of freedom to the code by which receptor gene choice and neuronal identities are specified in the olfactory system.

\section{Materials and Methods}

Drosophila stocks and transgenic flies. Stocks were cultured at $25^{\circ} \mathrm{C}$ in a humidified incubator. Flies used for electrophysiology were 5-10 d old. 


\section{Table 1. acj6 splice forms}

\begin{tabular}{lll}
\hline Splice form & Exons & Sources \\
\hline $\mathrm{A}^{a}$ & $1,2,3,4,51,6,7,81,9$ & MP \\
$\mathrm{C}^{b}$ & $1,2,4,5 \mathrm{~s}, 6,7,8 \mathrm{~s}, 9$ & Antenna \\
$\mathrm{D}^{b}$ & $1,2,3,4,51,6,7,8 \mathrm{~s}, 9$ & Larva, antenna \\
$\mathrm{E}^{b}$ & $1,2,4,51,6,7,8 \mathrm{~s}, 9$ & MP \\
$\mathrm{F}^{b}$ & $1,4,51,6,7,8 \mathrm{~s}, 9$ & Antenna, MP \\
$\mathrm{G}$ & $1,2,3,4,5 \mathrm{~s}, 6,7,8 \mathrm{~s}, 9$ & Antenna \\
$\mathrm{H}$ & $1,4,5 \mathrm{~s}, 6,7,8 \mathrm{~s}, 9$ & Larva, antenna, MP \\
$\mathrm{I}$ & $1,4,51,6,7,81,9$ & Larva, antenna, MP \\
$\mathrm{J}$ & $1,4,5 \mathrm{~s}, 6,7,81,9$ & Larva, antenna, MP \\
$\mathrm{K}$ & $1,2,4,5 \mathrm{~s}, 6,7,81,9$ & Antenna \\
$\mathrm{L}$ & $1,3,4,5 \mathrm{~s}, 6,7,8 \mathrm{~s}, 9$ & Antenna, MP \\
$\mathrm{M}^{b}$ & $1,3,4,5 l, 6,7,8 \mathrm{~s}, 9$ & Larva, antenna, MP \\
$\mathrm{N}$ & $1,3,4,5 \mathrm{~s}, 6,7,81,9$ & Larva \\
\hline
\end{tabular}

Thirteen acj6 splice forms were amplified in this study from maxillary palps (MP), antennae, or whole larvae. The exons contained in each splice form are indicated.

${ }^{a}$ Predicted by Flybase (http://flybase.org).

${ }^{b}$ Described by Certel et al. (2000).

(Invitrogen). The primer pair 5'-ATTAGCGGCCGCATGACAATGTCGATGTATTCGA-3' and 5'-ATTAGGTACCTCAGTATCCAAATCCCGCCGAAC-3' (Yale Keck Oligo Synthesis) was used to amplify acj6 transcripts from cDNA preparations. The PCR products were cloned into vector pGEM-Teasy (Promega) and sequenced.

acj6 cDNA sequences were verified, and sequences were subcloned into the pUAST vector (Brand and Perrimon, 1993). The structure we determined for acj6- $F$ varies from that reported by Certel et al. (2000), who referred to it as Acj6 $(1,4)$, in that we found that it contains exon 5 as a long form (5l) as opposed to exon 5 as a short form (5s).

For in situ hybridization, digoxigenin-labeled RNA probes were hybridized to whole maxillary palps attached to the proboscis. Probes were detected with alkaline phosphatase-conjugated anti-digoxigenin antibody as described previously (Goldman et al., 2005).

Electrophysiology. Odor stimuli were presented and action potentials were recorded as described previously (Goldman et al., 2005) by placing an electrode through the sensillum wall and in contact with the sensillum lymph that bathes the ORN dendrites. Odorants were diluted 1:100 in paraffin oil, and $50 \mathrm{ml}$ of the odorant dilutions were pipetted onto a filter paper disc (Waterman) placed inside a glass cartridge. During stimulation, odor vapor in the cartridge was puffed into an airstream that was directed at the fly head. The number of impulses during the $0.5 \mathrm{~s}$ stimulation period was counted, and the spontaneous impulse rate was subtracted from the response rate.

Data analysis. Hierarchical cluster analysis of neuronal responses was performed using PAST (version 1.82b) as described previously (Hallem and Carlson, 2006). Neurons were classified based on clustering of their responses profiles.

B

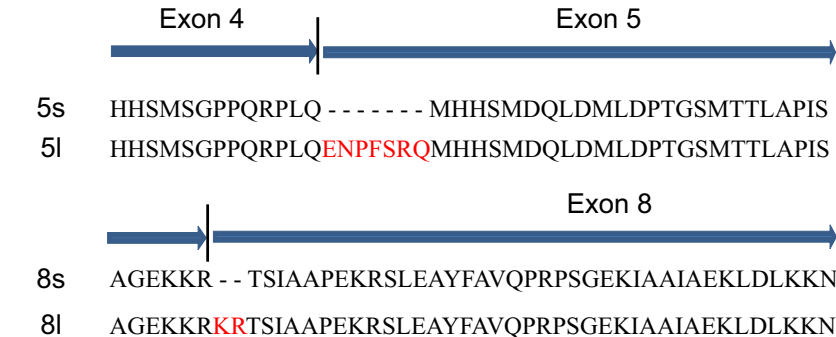

C

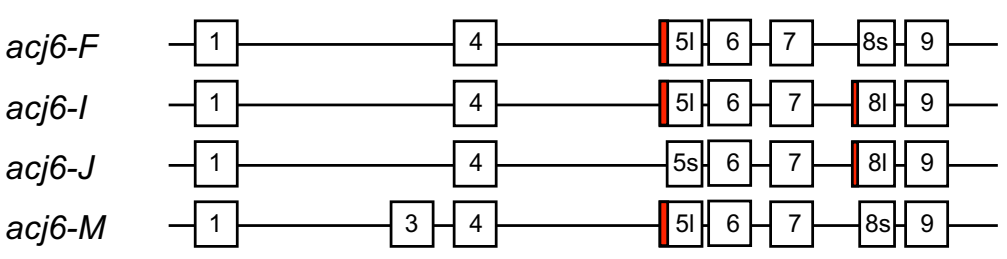

Figure 1. Alternative splice forms of acj6. $\boldsymbol{A}$, The exon structure of acj6 (not drawn to scale), showing four alternatively spliced exons. Exons 5 and 8 have alternative $5^{\prime}$ splice acceptor sites, and the variable portions of the exons are shown in red. The POU-specific domain (SD) and the POU homeodomain (HD), both shown to be involved in DNA binding of other POU proteins, are indicated. The POU IV box, a conserved N-terminal motif, consists of the underlined portions of exon 1 and exon 4. $\boldsymbol{B}$, The $5 \mathrm{I}$ form encodes 7 extra amino acids compared with the $5 \mathrm{~s}$ form. The 81 form is 2 aa longer than the $8 \mathrm{~s}$ form. $C$, Exon compositions of four individual acj6 cDNAs. Flies carrying each of these acj6 transgenes were generated.

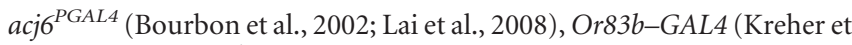
al., 2005), and acj6 6 (Clyne et al., 1999b) were described previously. All acj6 cDNA constructs were sequenced and injected into $w^{1118}$ embryos. For acj6-I and acj6-J, at least two independent transgenic lines were analyzed. acj6- $F$ and acj6- $M$ were described by Certel et al. (2000), who found no phenotypic variance between multiple transgenic lines of acj6- $F$ or acj6- $M$. acj6- $F$ and acj6- $M$ were referred to by Certel et al. (2000) as Acj6(1,4) and Acj6(1,3,4).

Molecular biology. Two hundred antennae and 600 maxillary palps of Canton-S wild-type flies were hand dissected, and total mRNAs were extracted separately with Qiagen RNeasy spin columns according to the protocol of the manufacturer. cDNAs were synthesized with a Superscript II kit

\section{Results \\ Multiple acj6 splice forms are expressed in the olfactory organs}

We identified 13 alternative splice forms of acj6 by reverse transcription (RT)-PCR analysis (Table 1, Fig. 1). Five of these splice forms were described previously by Certel et al. (2000), and another was predicted (http://flybase.org). Eight acj6 splice forms were found expressed in the antenna. In the maxillary palp, we identified eight splice forms, which overlap partially with the antennal cohort. From 600 hand-dissected maxillary palps, we isolated the most cDNAs for splice form acj6- $F$, followed by acj6- $M$, acj6- $J$, acj6- $H$, acj6-I, and acj6-A (supplemental Table 1, available at www.jneurosci.org as supplemental material). Splice forms acj6-E and acj6- $L$ were represented by a single clone. The larva also expresses multiple acj6 splice forms, and all but one of these, acj6- $N$, were also identified in the adult olfactory organs.

Of the nine exons of acj6, all splice forms contain exons 1, 4, 6, 7, and 9. Exons 2, 3, 5, and 8 are alternatively spliced, in either of two respects (Fig. $1 A, B$ ). Exons 2 or 3 can be included or excluded. The inclusion of either exon 2 or exon 3 disrupts a conserved $\mathrm{N}$-terminus motif, referred to as the POU IV box, which may mediate protein-protein interactions (Gerrero et al., 1993; Turner et al., 1994). Exons 5 and 8 are included in all transcripts but have alternative 5 ' ends; thus, exon 5 is included as either 51 or $5 \mathrm{~s}$, and exon 8 is also included in either a long or short form ( 81 or $8 \mathrm{~s})$. The $5 \mathrm{l}$ form is 7 aa longer than $5 \mathrm{~s}$, and $8 \mathrm{l}$ is 2 aa longer than $8 \mathrm{~s}$. 
A

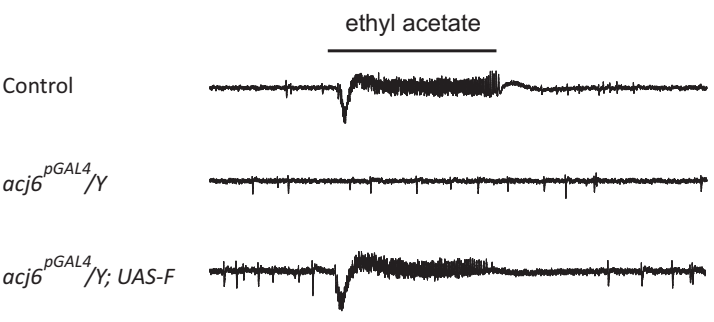

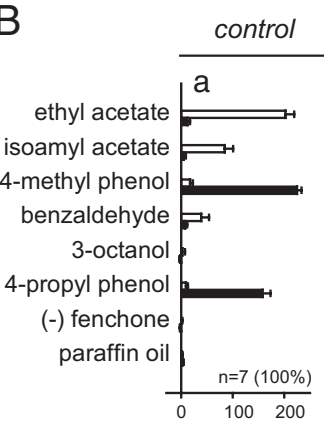

$(1 \mathrm{~A}, 1 \mathrm{~B})$

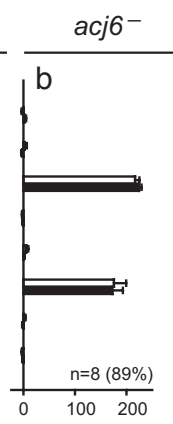

(1B, 1B)

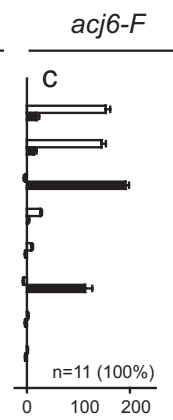

$(1 \mathrm{~A}, 1 \mathrm{~B})$

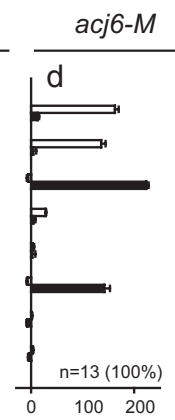

$(1 \mathrm{~A}, 1 \mathrm{~B})$

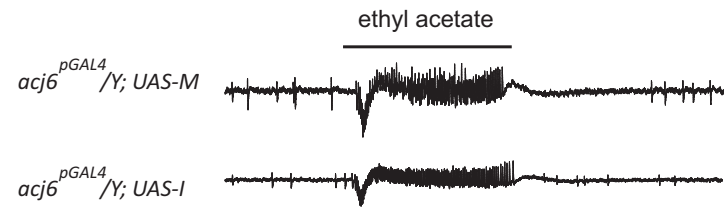

$\operatorname{acj} 6^{p G A L 4} / Y$; UAS-J

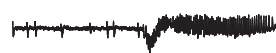

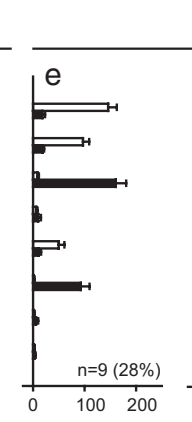

$(1 \mathrm{~A}, 1 \mathrm{~B})$

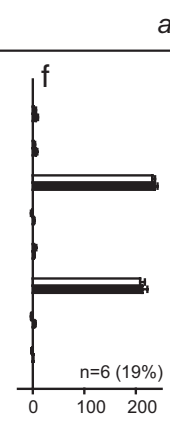

(1B, 1B)
acj6-I

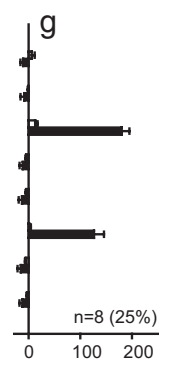

$(0,1 \mathrm{~B})$

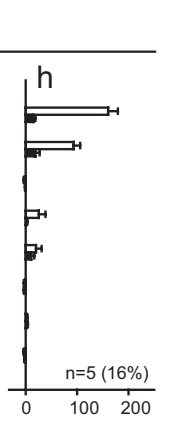

$(1 \mathrm{~A}, 0)$

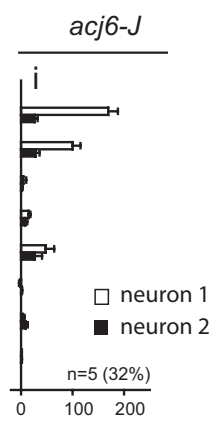

$(1 \mathrm{~A}, 0)$

ORN response (spikes/s)

Figure 2. Differential rescue of pb1 odor response by acj6 splice forms. $A$, Response to $0.5 \mathrm{~s}$ pulse (horizontal bar) of ethyl acetate was restored by expressing each of the four splice forms tested. Sample traces of 1.5 s recordings from each genotype are shown. acj6 ${ }^{P G A L 4}$ is a null allele of acj6 that expresses GAL4, which drives expression of UAS transgenes in maxillary palp 0RNs. The downward deflections in the traces shortly after stimulus onset reflect receptor potentials, which have not been filtered in these recordings. Action potentials from the two neurons housed in each sensillum can be observed and distinguished by amplitude; the larger-amplitude action potential comes from "neuron 1" (referred to as the A neuron in wild type), whereas the smaller-amplitude action potential comes from "neuron 2" (referred to as the B neuron in wild type). B, 0dor response profiles of pb1 ORNs in control, acj6 mutants, and flies expressing a single splice form of acj6. ORN responses to a panel of odorants were measured as the frequency of action potentials (spikes per second). Because there are differences in 0RN responses between wild-type strains (Goldman et al., 2005), we used $a c j 6^{P G A L 4} /+$ female flies as controls. We did not observe differences between male and female maxillary palps. acj6 $\sigma^{-}$is acj6 ${ }^{P G A L 4} / Y$. acj6- $F$, acj6-M, acj6-J, and acj6-I are acj6 ${ }^{P G A L 4} / Y_{\text {and }}$ contain one copy of the indicated transgene. $\boldsymbol{a}$, Control pb1 sensilla contain two ORNs with different response spectra, pb1A and pb1B (1A, 1B). $\boldsymbol{b}$, Most pb1 sensilla in acj6 ${ }^{-}$contain two 0RNs with the same response spectrum as the wild-type pb1B $(1 B, 1 B) . c, d$, Splice forms F and M fully restored pb1 sensilla to the wild-type configuration (1A, 1B). e-i, pb1 sensilla in flies expressing I or J splice forms were variable, and the major types are shown. Percentages indicate fractions of sensilla that contained either a pb1A or pb1B neuron. " 0 " indicates the lack of a responsive neuron; in some cases, we observe a neuron that produces spontaneous action potentials but is unresponsive, and, in some cases, there is no electrical evidence of a neighboring neuron. Error bars indicate SEM.

Alternative splicing of exon 5 does not affect the sequence of the POU IV box or the POU domain. The extra 2 aa of exon 8 lie within the POU homeodomain of Acj6, a region that has been shown in other POU proteins to be required for DNA binding (Sturm and Herr, 1988; Ingraham et al., 1990; Verrijzer et al., 1992b). Splice forms containing all possible exon 5 and exon 8 combinations were found.

\section{Construction of flies expressing a single acj6 splice form}

We examined the functions of individual acj6 splice forms by analyzing flies that express a single splice form in an acj6 background. Such flies were obtained by crossing flies containing UAS-acj6 transgenes, each corresponding to a different splice form, to acj ${ }^{P G A L 4}$ flies (Bourbon et al., 2002). acj6 ${ }^{P G A L 4}$ contains a GAL4 insertion that disrupts acj6 function and drives upstream activating sequence (UAS) reporter expression in all or almost all maxillary palp ORNs (supplemental Fig. S1, available at www. jneurosci.org as supplemental material). We selected four different splice forms for expression studies, including ones that included or excluded exon 3 , that included $5 \mathrm{l}$ or $5 \mathrm{~s}$, and that included 81 or $8 \mathrm{~s}$ (Fig. 1C). All of these splice forms (acj6-F, acj6-I, acj6- $M$, and acj6- $J$ ) were found to be expressed in the maxillary palp.

The function of each splice form was analyzed in the maxillary palp, which contains six ORN classes whose phenotypes can be measured quantitatively and precisely. In acj6 $6^{6}$, a null mutant, four of the six ORN classes are not found, but a new class emerges, pb2C, and one class of ORN, pb1B, is sometimes found dupli- cated within a sensillum (Clyne et al., 1999b), as described below. The acj $6^{\text {PGAL4 }}$ phenotype is the same as that of the null acj6 6 allele. We were interested in whether any of the four acj6 splice forms were able to function individually in the specification of ORN identity.

Individual splice forms can rescue pb1 but do so differentially Sensilla containing the normal pb1 configuration, consisting of a pb1A cell paired with a pb1B cell ( $\mathrm{pb} 1 \mathrm{~A}, \mathrm{pb} 1 \mathrm{~B})$, are not found in acj6 null mutants. pb1A cells are not observed; rather, most sensilla contain two pb1B cells that are paired in the same sensillum (pb1B, pb1B), and a smaller fraction of sensilla contain a normal pb1B cell that is not paired with a responsive neuron $(0, \mathrm{pb} 1 \mathrm{~B})$ (Clyne et al., 1999b).

We initially asked whether any of the four splice forms were able to rescue the loss of the pb1A neuron. We found that all four were able, when singly expressed in acj6 $6^{P G A L 4}$, to rescue the response of the pb1A neuron to ethyl acetate (Fig. $2 A$ ). Thus, a single splice form of acj6 is able to function individually in this context, and, despite their different structures, each of four tested splice forms retained this function. However, we observed that the frequencies at which the pb1A phenotype was rescued differed, which led us to conduct a more extensive analysis.

We measured the responses of pb1 sensilla to a diagnostic panel of odorants (Fig. $2 \mathrm{~B}$ ). In the wild type, all tested pb1 sensilla $(n=7)$ contain the expected pairing of a pb1A cell with a pb1B cell (pb1A, pb1B). In acj6 ${ }^{P G A L 4}$, we found results like those found in acj $^{6}$ : no (pb1A, pb1B) sensilla were observed, but there were 
A
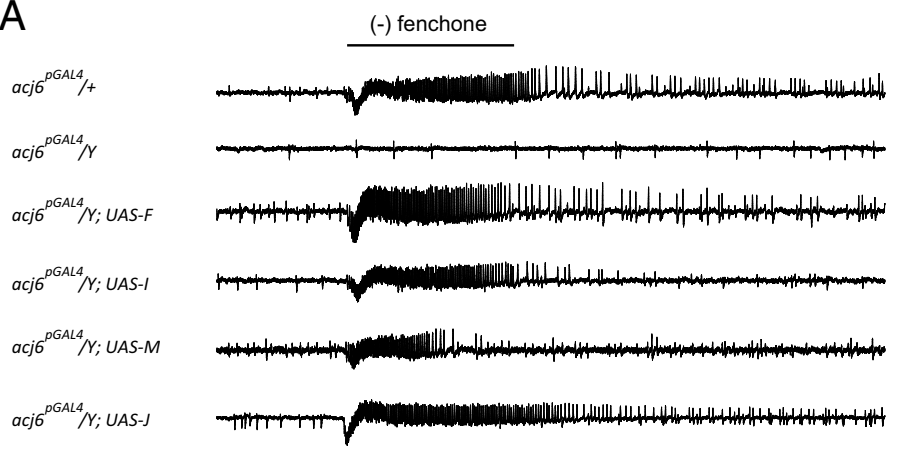

B

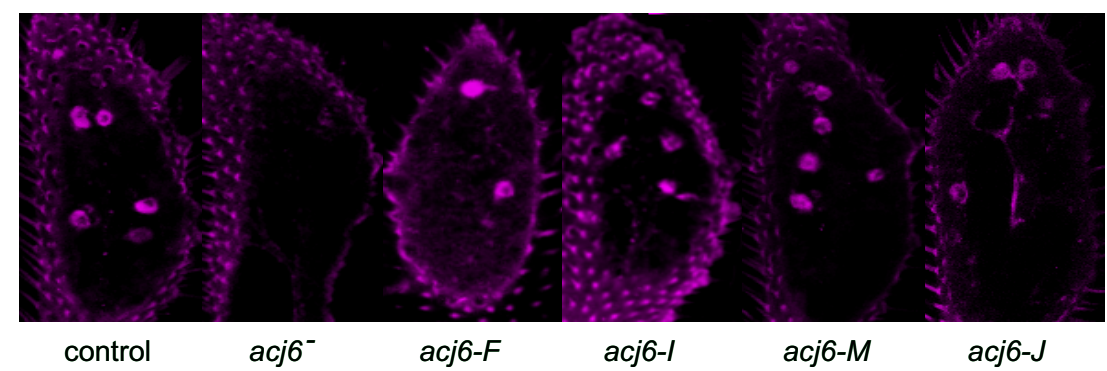

Figure 3. Multiple acj6 splice forms rescue pb2A neurons. $A$, The response of pb2A to (-)fenchone is restored by multiple splice forms. Sample traces of $2 \mathrm{~s}$ recordings from each genotype are shown. Scale bar represents the $500 \mathrm{~ms}$ odor stimulation period. $B$, The expression of the pb2A receptor gene Or85e is rescued by the expression of single splice forms, as shown by in situ hybridization of Or85e RNA probes to the maxillary palp. Autofluorescent cuticle can be seen at the periphery of each section.

sensilla containing a pb1B neuron that is either paired with another pb1B neuron (pb1B, pb1B) $(n=8)$ (Fig. $2 \mathrm{Bb})$ or that is not paired with a responsive neuron $(0, \mathrm{pb} 1 \mathrm{~B})(n=1$; data not shown).

When the acj6-F splice form was expressed in this acj6 background, it fully rescued the pb1 phenotype, yielding (pb1A, pb1B) sensilla that responded as expected to all odorants of the panel (Fig. $2 B c$ ). The same full rescue was observed with acj6-M (Fig. $2 B d)$. Both of these splice forms contain 51 and 8 s exons. No abnormal sensilla containing either pb1A or pb1B cells were observed.

When the acj6-I splice form was expressed, a more complex pattern emerged. This splice form was capable of fully rescuing the pb1 phenotype, but, of 32 sensilla containing pb1A or pb1B cells, only $28 \%$ were of the (pb1A, pb1B) type (Fig. 2 Be). Another $19 \%$ were of the (pb1B, pb1B) type (Fig. $2 B f$ ), and $25 \%$ of the sensilla were of the (0, pb1B) type (Fig. $2 \mathrm{Bg}$ ). Surprisingly, we also found five sensilla $(16 \%)$ that were of a type not seen in either wild type or acj6: (pb1A, 0) (Fig. 2Bh). The absence of pb1B was unexpected, because pb1B cells are present in acj6 null mutants and were therefore thought to be specified independently of acj6 (Fig. $2 \mathrm{Bb}$ ) (Clyne et al., 1999a). In addition, a few sensilla appeared to contain either a pb1A cell or a pb1B cell paired with other cell types (data not shown).

Expression of the acj6- $J$ splice form again revealed the unexpected (pb1A, 0) type (Fig. $2 \mathrm{Bi}$ ), at an even higher frequency: $32 \%$ of 19 sensilla that contained either pb1A or pb1B. Only $5 \%$ of sensilla showed a complete rescue of the (pb1A, pb1B) phenotype. Another $37 \%$ of sensilla contained a neuron with the odor specificity of pb1A paired with a neuron other than pb1B (data not shown). These other neurons were of various odor specificities, and, in two sensilla, the pb1A cell was paired with a novel ORN class that we have termed pb1C and that will be shown and considered further below. The remaining 31\% of sensilla ap- peared to contain a pb1B neuron paired with another pb1B, with an unresponsive neuron, or with a non-pb1A neuron.

In summary, all tested acj6 splice forms were able to function independently in $\mathrm{pb} 1$, in that all rescued at least some pb1A cells. However, the number of normal (pb1A, pb1B) sensilla varied dramatically with different splice forms, and some splice forms gave rise to the $(0, \mathrm{pb} 1 \mathrm{~B})$ and novel (pb1A, 0) types. We note finally for comparison that, in a survey of 232 sensilla in the wild-type maxillary palp, only $3 \%$ of sensilla contained a single functional cell, and only $1 \%$ showed a noncanonical combination of cell types (de Bruyne et al., 1999).

\section{Individual splice forms act both positively and negatively in $\mathrm{pb} 2$} pb2 sensilla in wild type contain a (pb2A, pb2B) pair. pb2A coexpresses two $\mathrm{Or}$ genes, Or85e and Or33c, and is unique among maxillary palp ORNs in that it shows a strong response to $(-)$ fenchone. The strong fenchone response has been shown previously to be conferred by Or85e (Goldman et al., 2005). pb2B expresses Or46a and responds strongly to 4-methyl phenol. In $a c j 6^{6}$ and $a c j 6^{\text {PGAL4 }}$, neither a pb2A nor a $\mathrm{pb} 2 \mathrm{~B}$ cell is found; rather, a new type of sensillum emerges that contains a novel ORN class, pb2C (Clyne et al., 1999b). pb2C is not paired with a responsive neuron in this sensillum (pb2C, 0 ).

All four acj6 splice forms, despite their structural differences, were able to induce a fenchone response in an acj6 ${ }^{P G A L 4}$ background (Fig. 3A). The simplest interpretation of this result is that each is able to induce expression of the Or85e gene. We confirmed this interpretation by in situ hybridization of an Or85e probe to maxillary palps expressing acj6-F, acj6-I, acj6- $M$, and acj6-J (Fig. 3B).

Although all splice forms appeared capable of rescuing the odorresponse spectrum of the pb2A neuron, only one splice form, acj6- $J$, induced normal (pb2A, pb2B) sensilla (Fig. $4 A, B, E$ ), and, even in this case, only $42 \%$ of sensilla containing pb2A or pb2B ORNs were of the (pb2A, pb2B) type. All splice forms suppressed the $(\mathrm{pb} 2 \mathrm{C}, 0)$ type that is normally observed in acj6 mutants: of a total of 65 sensilla expressing the four splice forms, only one was of this type, in a fly expressing acj6-I. We note with interest the observation that acj6-J, but not acj6- $F$ or acj6- $M$, was able to induce normal ( $\mathrm{pb} 2 \mathrm{~A}, \mathrm{pb} 2 \mathrm{~B})$ sensilla; in the case of $\mathrm{pb} 1$, the reciprocal results were obtained.

acj6- $F$ and acj6- $M$ were similar in that both induced two unexpected types of sensilla (Fig. $4 C, D, G, H$ ). One type contained a normal $\mathrm{pb} 2 \mathrm{~B}$ cell and a novel ORN that responded to 3 -octanol but to no other odorants of the panel (Fig. 4C,D). We term this ORN pb2D and consider its origin below. This (pb2D, pb2B) sensillum represented 25 and $21 \%$ of the sensilla containing pb2A or pb2B neurons in flies expressing acj6- $F$ and acj6- $M$, respectively. The second unexpected type, representing 45 and 53\% of the pb2 sensilla in acj6- $F$ - and acj6- $M$-expressing flies, contained a normal pb2B cell and a novel ORN that responded to both 3-octanol and fenchone and that we term pb2E (Fig. 4G,H). We note that flies expressing acj6- $J$ also contained pb2E cells: in four cases (33\%), the 


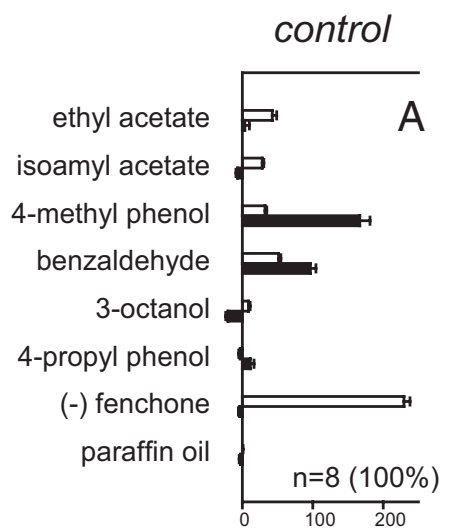

(2A, 2B)

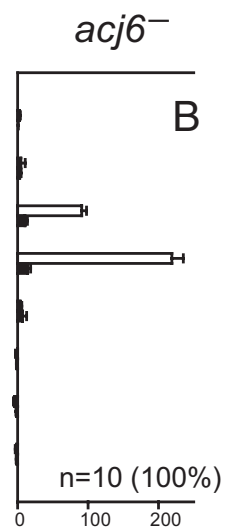

$(2 \mathrm{C}, 0)$

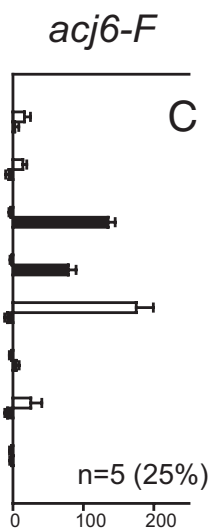

(2D, 2B)

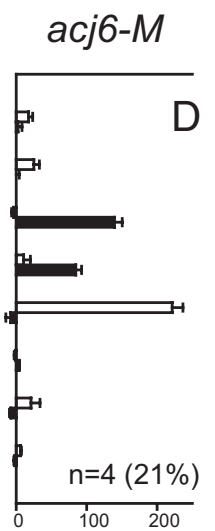

(2D, 2B)

ORN response (spikes/s)

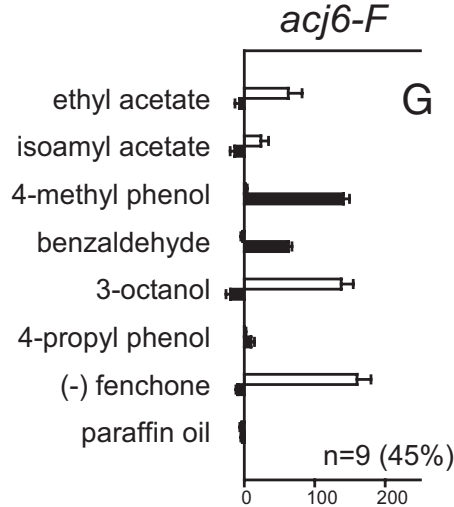

(2E, 2B)

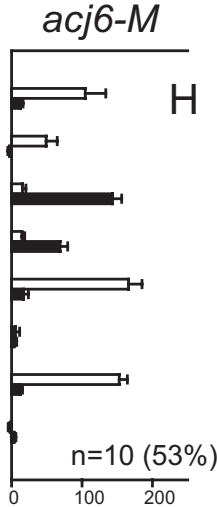

(2E, 2B)

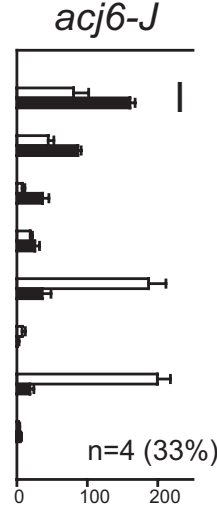

$(2 \mathrm{E}, 1 \mathrm{~A})$

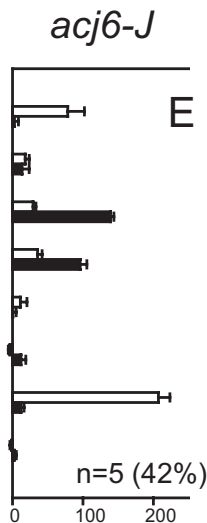

$(2 \mathrm{~A}, 2 \mathrm{~B})$
acj6-I

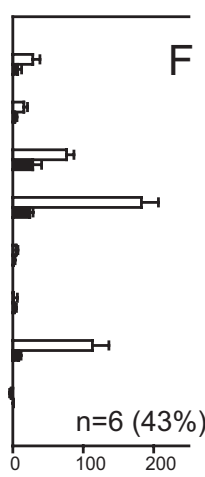

$(2 \mathrm{~F}, 0)$

ORN response (spikes/s)

Figure 4. Partial rescue of pb2 sensilla by individual acj6 splice forms. Odor response profiles of $0 R N s$ in pb2 sensilla. $A$, Wild-type pb2 sensilla contain two ORNs, pb2A and pb2B (2A, 2B). $B$, pb2 sensilla in acj6 maxillary palps do not contain ORNs with the spectra of either pb2A or pb2B but instead contain a distinct ORN (pb2C; white bars). These sensilla $(2 C, 0)$ either contain a second neuron that does not respond to any tested odorant, or they do not contain a second neuron that shows electrical activity. C-I, Diverse sensillum types were identified in flies expressing single acj6 splice forms. Only the major types are shown. Many neurons with noncanonical response profiles were classified and designated as 2D, 2E, and 2F. Black bars represent the 0RNs with larger amplitudes; white bars indicate ORNs with smaller amplitudes. Percentages indicate fractions of sensilla that contained an ORN responding to (-)fenchone. Error bars indicate SEM.

pb2E cell was paired with pb1A (Fig. 4I), and in two cases (13\%), it was paired with unidentified cells (data not shown).

Expression of acj6-I gave rise to a number of sensillum types of which the most frequent $(43 \%)$ contained a novel neuron, pb2F, paired with an unresponsive neuron (Fig. $4 F$ ). pb2B neurons were rarely observed in acj6-I-expressing flies.

In summary, all tested splice forms appear able to induce expression of the Or85e receptor, which underlies response to fenchone in $\mathrm{pb} 2 \mathrm{~A}$, and all appear to suppress formation of the $\mathrm{pb} 2 \mathrm{C}$ neuron that appears in the absence of acj6 expression. These results suggest that an individual splice form can act as both an activator and a repressor. pb2B neurons are present in most sensilla expressing acj6-F or acj6-M splice forms but were rarely observed in sensilla expressing acj6-I. Expression of some splice forms yielded novel neuron classes that are analyzed further below. None of the splice forms completely rescued $\mathrm{pb} 2 \mathrm{~A}$ or $\mathrm{pb} 2 \mathrm{~B}$.

\section{Rescue of $\mathrm{pb} 3$ occurs more frequently than rescue of $\mathrm{pb} 2$}

Unlike pb2, pb3 sensilla were rescued by all splice forms (Fig. 5A). Moreover, the (pb3A, pb3B) type was observed in $70-86 \%$ of sensilla with a pb3A or pb3B ORN. These figures are much higher than the corresponding figures for $\mathrm{pb} 1$ or $\mathrm{pb} 2$ sensilla, with the exception of the rescue of pb1 by acj6-F (100\%) and acj6-M
$(100 \%)$. The rescue of the pb3A neuron was confirmed by in situ hybridization with $O r 59 c$, the receptor gene that underlies response of pb3A, in two cases (Fig. 5B).

\section{Individual splice forms specify maxillary palp ORNs with} response spectra of larval odor receptors

What is the origin of the novel ORNs that were induced by expression of single splice forms of acj6? We were interested in how an alteration in the expression of acj6 might lead to these changes in odor specificities, which presumably result from changes in odor receptor gene choice.

pb1C was observed paired with pb1A after expression of acj6-J; it was also observed paired with another, unidentified cell in this genotype (data not shown). We noticed that its response profile closely resembled the response profile of a larval odor receptor that had been analyzed in the course of a systematic study of the larval Or repertoire (Kreher et al., 2005, 2008). In this analysis, the 25 Or genes that are expressed in the larva were each functionally expressed in an in vivo expression system, the "empty neuron." Briefly, each larval Or gene was expressed in a mutant antennal ORN that lacks an endogenous functional receptor, and the odor response spectrum that was conferred by each larval Or gene was measured by single-unit electrophysiol- 

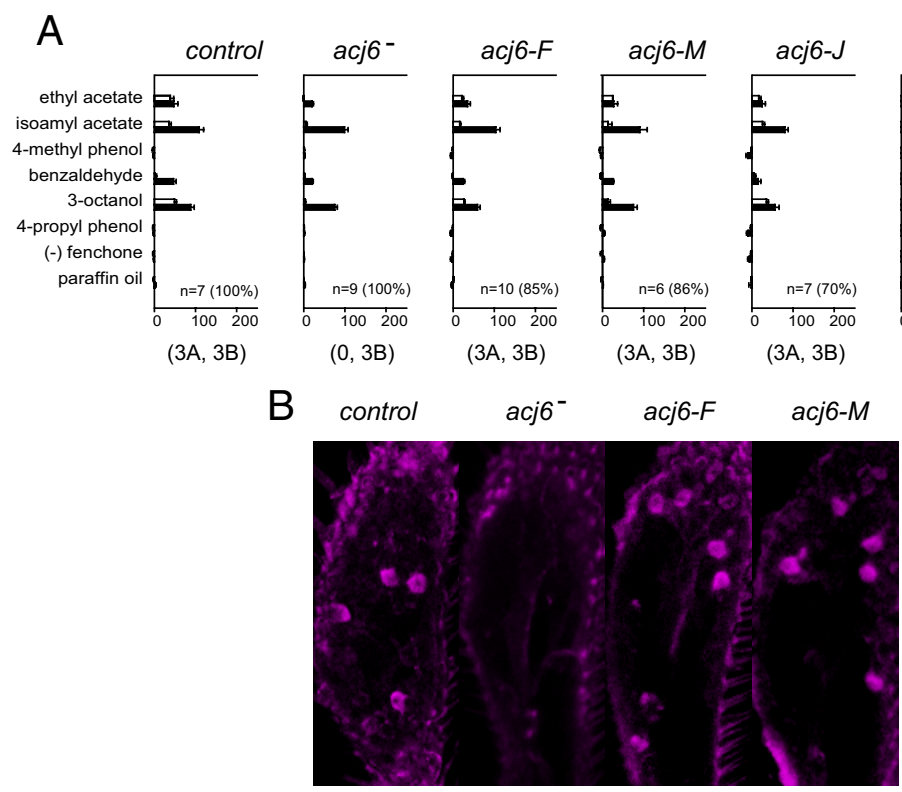

Figure 5. The $\mathrm{pb} 3$ sensilla are rescued by four splice forms. $A, O R N$ response profiles of $p b 3$ sensilla. When multiple forms of $\mathrm{pb} 3$ were present, only the major types are shown. Error bars indicate SEM. B, Expression of Or 59 c, the receptor gene expressed in pb3A of wild type, was rescued by the expression of the indicated splice forms, as shown by in situ hybridization of $0 \mathrm{r} 59$ c RNA probes to the maxillary palp.

ogy. One larval receptor, Or85c, gave a response spectrum that matched that of pb1C (Fig. 6A). Moreover, both Or85c and pb1C shared another characteristic: a remarkably long-lasting response to 2-heptanone (Fig. 6B). The simplest interpretation of these results is that pb1C arises from the ectopic expression in the maxillary palp of the larval $0 r 85 c$ gene.

pb2D was observed in both acj6- $F$ and acj6- $M$ and responded only to 3-octanol among the odorants tested. This response agreed well with the response of another receptor, Or13a, which is expressed in the larval olfactory system as well as the antenna in wild type (Fig. 6C).

$\mathrm{pb} 2 \mathrm{E}$ has a fenchone response that resembles that of $\mathrm{pb} 2 \mathrm{~A}$, but it also has a 3-octanol response that is characteristic of $\mathrm{pb} 2 \mathrm{D}$. One possible interpretation of $\mathrm{pb} 2 \mathrm{E}$ is that it represents a hybrid of $\mathrm{pb} 2 \mathrm{~A}$ and $\mathrm{pb} 2 \mathrm{D}$ and that its fenchone and 3-octanol responses derive from the expression of Or85e and Or13a, respectively. As a test of this possibility, we compared the responses of an Or13aexpressing $\mathrm{ORN}, \mathrm{pb} 2 \mathrm{~A}$, and $\mathrm{pb} 2 \mathrm{E}$ and found that the pb2E profile resembles the sum of the other two profiles, in support of this interpretation (Fig. 6D).

\section{Rescue of ORN identity by late expression of acj6}

Little is known about when acj6 acts in the process of receptor gene choice and the specification of ORN identity. acj6 has been shown to act in ORN axon targeting, which occurs primarily before the onset of Or gene expression (Clyne et al., 1999a; Jhaveri et al., 2000; Jefferis et al., 2004; Komiyama et al., 2004). However, it is not clear whether the specification of ORN classes also requires acj6 at an early stage. We asked whether it was required early by supplying it late: in an acj6 background, we expressed acj6- $F$ and acj6-M using an Or83b-GAL4 driver (Kreher et al., 2005). Or83b-GAL4 is expressed in all maxillary palp ORNs, but a green fluorescent protein reporter driven by $\mathrm{Or} 83 b-G A L 4$ is not detected until $\sim 82 \mathrm{~h}$ after puparium formation (APF) (Larsson et al., 2004; Ge et al., 2006). In contrast, Acj6 can be detected in the

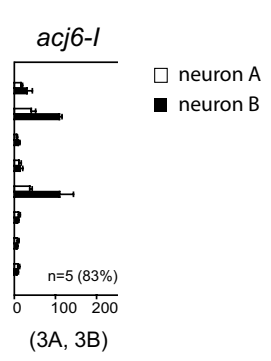

wild-type maxillary palp at $31 \mathrm{~h} \mathrm{APF} \mathrm{in}$ some cells and in most ORNs by $50 \mathrm{~h}$ (Clyne et al., 1999b; Komiyama et al., 2004).

We found that late expression of either acj6- $F$ or acj6- $M$ produced normal pb1A, pb1B, pb2B, pb3A, and pb3B cells (Fig. 7). Rather than pb2A cells, late expression of either splice form gave rise to pb2E cells (the cells that are very similar to pb2A except that they may express Or13a in addition to the endogenous Or85e receptor) (Fig. 4G,H), as occurred at lower frequencies when these splice forms were expressed earlier under the control of the acj6 driver. Interestingly, these six ORN classes were the only ones observed ( $n=$ 50 sensilla examined); no pb2A, pb2C, or pb2D cells were observed.

These results suggest that acj6 is not required early in ORN development for receptor gene choice in most ORNs but that it is capable of acting late.

\section{Discussion}

We have provided evidence that acj6 is spliced in at least eight different ways in the maxillary palp, potentially producing eight structurally distinct proteins. Different splice forms are functionally distinct, and different ORNs differ in their requirements for acj6 splice forms. Individual splice forms are capable of acting both positively and negatively in regulating the expression of odor receptor genes. It has been shown previously that odor receptor gene choice depends on a combinatorial code of transcription factors; the results presented here provide evidence that the process also depends on a multiplicity of splice forms. Thus, alternative splicing may expand the combinatorial code that underlies the process of receptor gene choice and the generation of ORN diversity.

\section{Functions of Acj6 splice forms in odor receptor gene choice}

Our results show that different splice forms that are expressed in the maxillary palp are not functionally equivalent in maxillary palp ORN specification. This finding is in agreement with a study showing that two acj6 splice forms produced different phenotypes when misexpressed in embryonic motor neurons, which do not normally express acj6 (Certel et al., 2000). The distinct functions of Acj6 variants are also consistent with results demonstrating that the mammalian POU genes Brn $3 a$ and Brn3b, which are highly homologous to each other and are mammalian orthologs of acj6, have distinct roles in the programming of retinal ganglion cell diversity (Badea et al., 2009).

We have found that a single splice form of acj6 is capable of full rescue of the odor response profile of some ORNs, such as the full rescue of pb1A by acj6-F (Fig. 2 B). This finding argues against a model in which Acj6 functions as an obligate heterodimer of splice forms. The ability of a single splice form to function is consistent with structural data indicating that the POU domain protein Oct-1 binds to DNA as a monomer and that Pit-1 can bind as a homodimer (Ingraham et al., 1990; Klemm et al., 1994; Jacobson et al., 1997).

Individual splice forms are able to act both positively and negatively. pb2A, which expresses Or85e, is not observed in acj6 
A

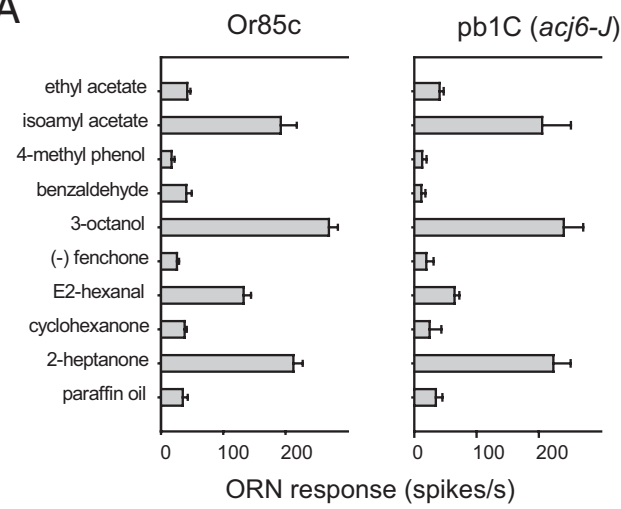

B

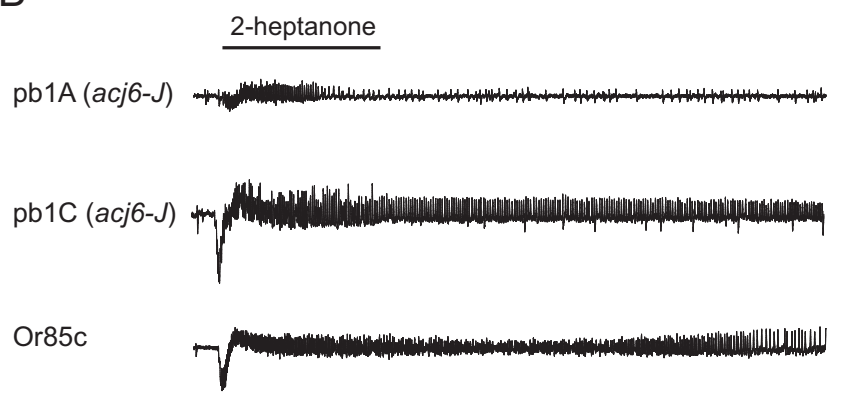

C
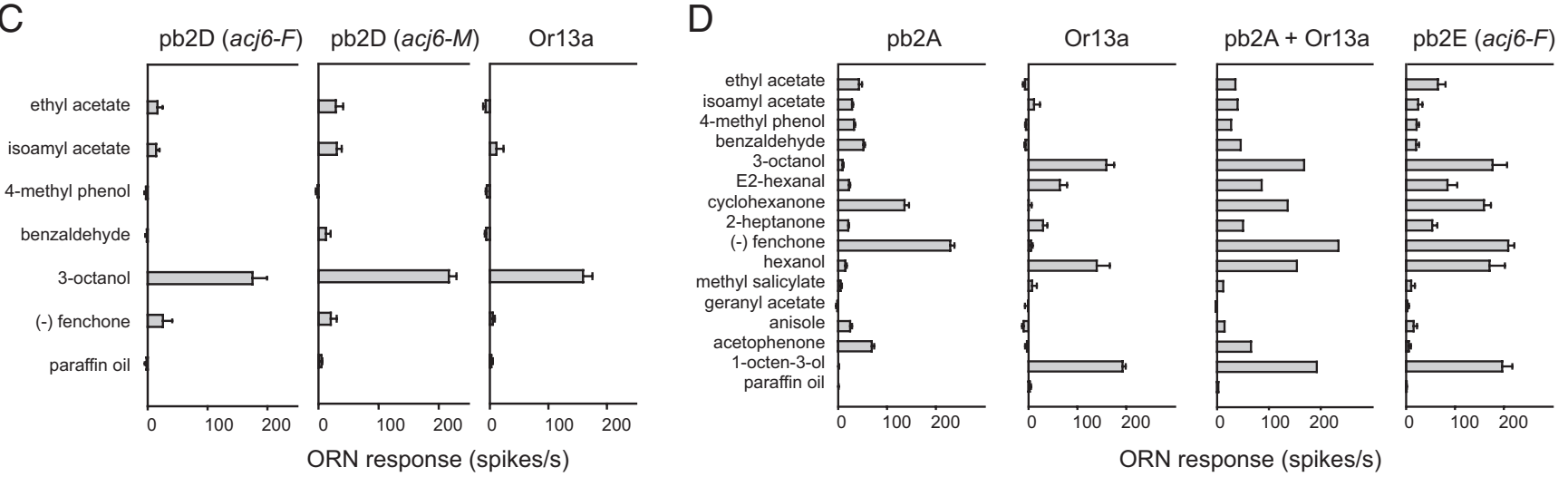

Figure 6. Novel ORNs with response profiles of larval odor receptors. $A$, The response profile of the larval receptor $0 \mathrm{r} 85 \mathrm{c}$ closely resembles that of pb1C. For Or85c, expressed in the empty neuron system, $n=12$; for pb1C, from flies expressing acj6-J, $n=3$. Data for Or85c are from Kreher et al. (2005). $\boldsymbol{B}$, The response termination dynamics of pb1C resemble those of Or85c. Sample $2 \mathrm{~s}$ recordings are shown. The $0.5 \mathrm{~s}$ stimulation period is indicated by the horizontal bar. A short-lasting response of pb1A is shown for comparison. The downward deflections in the traces shortly after stimulus onset reflect receptor potentials, which have not been filtered in these recordings. C, pb2D, from flies expressing acj6-F(n=5) or acj6-M $(n=4)$, has a similar response profile to that of the 0 r13a receptor $(n=5)$. The acj6-Fand acj6-M data are taken from Figure 4. $D$, The response profile of pb2E was compared with those of the wild-type pb2A ORN and Or13a expressed in the empty neuron system. The pb2E responses appear very similar to the linear summation of wild-type pb2A and Or13a responses (3rd panel from left). Error bars indicate SEM.

null mutants, whereas a novel pb2C cell appears as a result of the ectopic expression of Or45b (Clyne et al., 1999b; Bai et al., 2009). acj6- $F$, acj6- $M$, and acj6- $J$ are able to activate the expression of Or85e and repress the expression of Or45b. These results argue against a model in which activation is mediated uniquely by one subset of splice forms and repression is mediated uniquely by a complementary subset.

None of the splice forms fully rescued either pb2 or pb3 sensilla, although two of these splice forms fully rescued pb1. The simplest interpretation of these results is that ORNs differ in their requirements for acj6 splice forms. One explanation for the lack of full rescue is that some ORNs may require more than a single acj6 splice form. We cannot exclude the possibility that an untested splice form might fully rescue pb2 or pb3. However, we note that the number of splice forms identified in the maxillary palp (eight) exceeds the number of ORN classes (six), suggesting that some ORNs express more than one form. Single-cell RNA profiling may allow a determination of how many splice forms are expressed in each ORN class. We note with interest that there is ample precedent for heterodimerization of different POU proteins (Kemler et al., 1989; Voss et al., 1991; Verrijzer et al., 1992a; Vigano and Staudt, 1996). Heterodimerization of Acj6 isoforms could provide yet another level of functional complexity: in principle, eight Acj6 isoforms could form 28 distinguishable heterodimers.

Expression of a single splice form in some cases led to novel ORN fates, including pb1C, pb2D, and pb2E. Each of these fates is likely to arise from the ectopic expression of a larval Or gene. pb2D and pb2E have not been described previously; interestingly, a neuron with an odor response spectrum like that of pb1C was observed recently when a cDNA encoding $p d m 3$ was expressed in the maxillary palp. Or $85 c$ was found to be ectopically expressed as well, supporting our interpretation that $\mathrm{pb} 1 \mathrm{C}$ derives from the misexpression of this gene (Fig. 6A). pdm3 is a POU gene that genetically interacts with acj6 in the activation of Or $42 a$ (Tichy et al., 2008), which confers the response of pb1A. Perhaps certain forms of the two POU proteins cooperate in misexpressing Or85c. The occurrence of pb1C in flies expressing acj6-J, but not other splice forms, could reflect differential interactions between Acj6 isoforms and other transcription factors, perhaps expanding the capacity of the combinatorial code to specify odor receptor gene choice.

\section{acj6 splice forms: structure and function}

There are several degrees of freedom in the splicing of acj6. Exons 2 and 3 can each be included or excluded, and exons 5 and 8 can each be included in either long or short forms. If all four of these splicing events occur independently, $2^{4}=16$ splice forms could be produced. We have identified 13 of these 16 forms in extensive but not exhaustive RT-PCR analysis.

Inclusion of either exon 2 or 3 disrupts the POU IV box, which is believed to mediate protein-protein interactions (Gerrero et al., 1993; Xiang et al., 1993; Turner et al., 1994). acj6-M, which contains exon 3, conferred complete rescue of pb1A. These results 
suggest that the POU IV box is not required for all acj6 functions. Moreover, acj6- $F$ and acj6- $M$, which differ only in the presence or absence of exon 3 , conferred very similar or identical phenotypes in all tests in this study. Interestingly, $\mathrm{Brn}-3 a$ and $B r n-3 b$, mammalian orthologs of acj6, also undergo alternative splicing to generate transcripts that either include or lack the POU IV box (Theil et al., 1993, 1994; Xiang et al., 1993).

In contrast, acj6-I and acj6- $J$ functioned differently from each other and from acj6-F or acj6-M (Fig. 4). acj6-I and acj6- $J$ differ from each other only in the form of exon 5 that they contain. acj6-I and acj6- $F$ differ from each other only in the form of exon 8 that they contain. The simplest interpretation of these results is that functional differences are conferred by the inclusion of extra amino acids in exons 5 and 8 . The 2 aa that differ between exons 8 s and 81 are conserved in Caenorhabditis elegans and mammalian orthologs of Acj6, and they are located in the POU homeodomain. One possibility is that the exclusion of these amino acids alters the DNAbinding properties of Acj6; alternative splicing of other transcription factors has been shown previously to affect DNA sequence discrimination (Gogos et al., 1992; Hsu et al., 1992).

\section{Acj6 can act late}

We found that acj6 is capable of acting late in development. When driven by a lateacting promoter, acj6 splice forms were able to rescue pb1A, pb2B, and pb3A cells. The ability of acj6 to act late is consistent with a direct role in Or gene regulation, which in turn is consistent with the finding that Acj6 binds directly to several Or promoters (Bai et al., 2009). We note that studies with a temperature-sensitive allele of lozenge, which encodes a Runx domain-containing transcription factor, showed that it could modulate expression of Or genes after eclosion (Ray et al., 2007). These results, taken together, are of special interest in light of recent findings that there is plasticity in the expression of some chemoreceptor genes. For example, Or $59 b$ was found to be expressed at higher levels in old males than in young males (Zhou et al., 2009).

\section{Concluding remarks}

The process of odor receptor gene choice in Drosophila relies on a combinatorial code of transcription factors, which act in concert in the olfactory system to activate individual odor receptors while repressing all the others in individual ORNs. In this study, we have examined one of these transcription factors in detail and found that it is expressed in a variety of functionally distinct forms in the maxillary palp via alternative splicing. This variety expands the combinatorial code of receptor gene choice by adding another level of complexity. In this manner, the number of transcription factors needed to govern the selection of odor receptor genes may be reduced. Moreover, the efficiency and flexibility of this

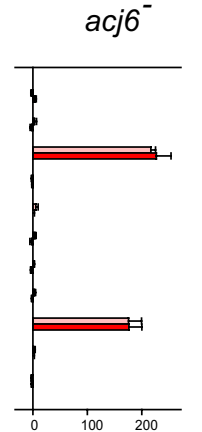

acj6-F

acj6-M
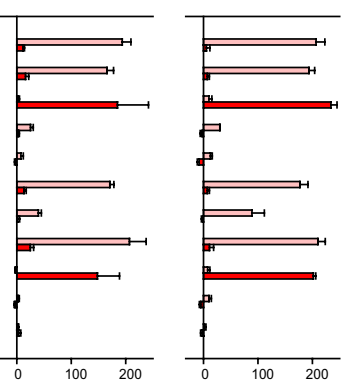

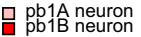
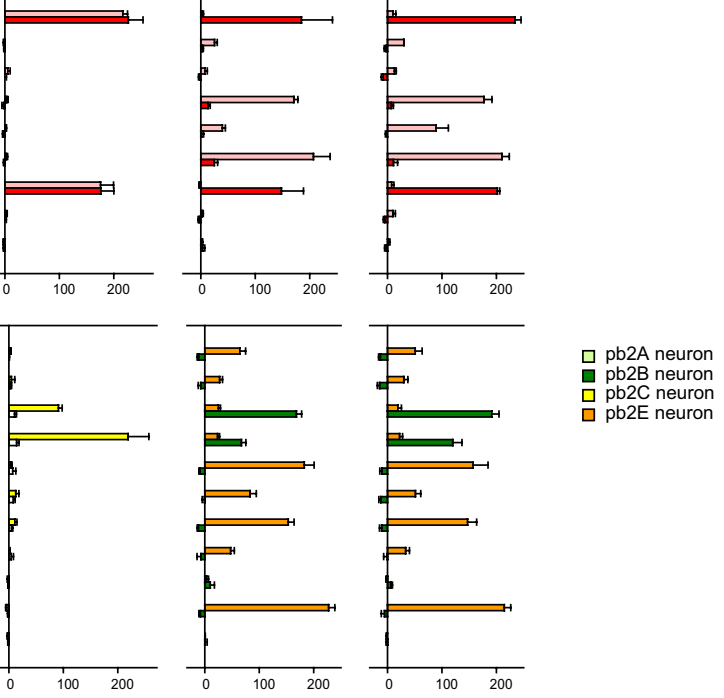

口 pb2A neuron pb2B neuron $\mathrm{p} p 2 \mathrm{C}$ neuron
$\mathrm{pb} 2 \mathrm{E}$ neuron
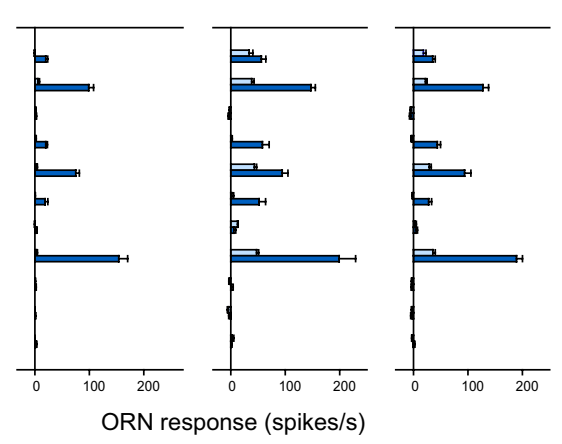
pb3A neuron

Figure 7. Rescue of ORN identity by late expression of acj6. ORN response profiles of control flies, acj6 flies, and flies expressing the indicated acj6 splice forms under the control of the Or83b-GAL4 driver. Control flies are acj6 $6^{\text {GALL }} /+$. The acj6 flies are $a j 6^{P G A L 4} / Y$. Control data are taken from Figures $2 A, 4 A$, and 5 , leftmost panel. acj6-Fis acj6 $/ Y$; Or83b-GAL4/+; UAS-acj6-F/+, and $a c j 6-M$ is $a c j 66^{6} / Y ; O r 83 b-G A L 4 /+;$ UAS-acj6-M. $5 \leq n \leq 12$. Note that, in pb2 sensilla, the acj6 mutation or single splice form expression generate 0 RNs with novel response profiles. Error bars indicate SEM.

regulatory system may be increased by the availability of an additional level at which natural selection can operate.

In addition to the problem of receptor gene choice, the olfactory system also faces the challenge of wiring specificity in the brain. Each ORN sends an axon to a particular destination in the antennal lobe of the brain, in which it forms connections with projection neurons. acj6 plays a role in this axonal targeting (Komiyama et al., 2004), and it will be interesting to determine whether alternative splicing of acj6 adds a degree of freedom to this process as well. Moreover, acj6 is required for dendritic targeting of projection neurons, and a single splice form, acj6- $F$, when individually expressed in acj6 flies, rescued some of the acj6 dendritic targeting defects (Komiyama et al., 2003). Future studies may determine whether different acj6 splice forms play distinct roles in the regulation of cell adhesion molecules, for example, and thereby act in the differential selection of targets by different neurons.

\section{References}

Badea TC, Cahill H, Ecker J, Hattar S, Nathans J (2009) Distinct roles of transcription factors brn3a and brn3b in controlling the development, morphology, and function of retinal ganglion cells. Neuron 61:852-864.

Bai L, Goldman AL, Carlson JR (2009) Positive and negative regulation of odor receptor gene choice in Drosophila by acj6. J Neurosci 29:1294012947. 
Bourbon HM, Gonzy-Treboul G, Peronnet F, Alin MF, Ardourel C, Benassayag C, Cribbs D, Deutsch J, Ferrer P, Haenlin M, Lepesant JA, Noselli S, Vincent A (2002) A P-insertion screen identifying novel X-linked essential genes in Drosophila. Mech Dev 110:71-83.

Brand AH, Perrimon N (1993) Targeted gene expression as a means of altering cell fates and generating dominant phenotypes. Development 118:401-415.

Certel SJ, Clyne PJ, Carlson JR, Johnson WA (2000) Regulation of central neuron synaptic targeting by the Drosophila POU protein, Acj6. Development 127:2395-2405.

Clyne PJ, Warr CG, Freeman MR, Lessing D, Kim J, Carlson JR (1999a) A novel family of divergent seven-transmembrane proteins: candidate odorant receptors in Drosophila. Neuron 22:327-338.

Clyne PJ, Certel SJ, de Bruyne M, Zaslavsky L, Johnson WA, Carlson JR (1999b) The odor specificities of a subset of olfactory receptor neurons are governed by Acj6, a POU-domain transcription factor. Neuron 22:339-347.

Couto A, Alenius M, Dickson BJ (2005) Molecular, anatomical, and functional organization of the Drosophila olfactory system. Curr Biol 15: 1535-1547.

de Bruyne M, Clyne PJ, Carlson JR (1999) Odor coding in a model olfactory organ: the Drosophila maxillary palp. J Neurosci 19:4520-4532.

de Bruyne M, Foster K, Carlson JR (2001) Odor coding in the Drosophila antenna. Neuron 30:537-552.

Fishilevich E, Domingos AI, Asahina K, Naef F, Vosshall LB, Louis M (2005) Chemotaxis behavior mediated by single larval olfactory neurons in Drosophila. Curr Biol 15:2086-2096.

Fuss SH, Ray A (2009) Mechanisms of odorant receptor gene choice in Drosophila and vertebrates. Mol Cell Neurosci 41:101-112.

Ge H, Krishnan P, Liu L, Krishnan B, Davis RL, Hardin PE, Roman G (2006) A Drosophila nonvisual arrestin is required for the maintenance of olfactory sensitivity. Chem Senses 31:49-62.

Gerrero MR, McEvilly RJ, Turner E, Lin CR, O'Connell S, Jenne KJ, Hobbs MV, Rosenfeld MG (1993) Brn-3.0: a POU-domain protein expressed in the sensory, immune, and endocrine systems that functions on elements distinct from known octamer motifs. Proc Natl Acad Sci U S A 90:10841-10845.

Gogos JA, Hsu T, Bolton J, Kafatos FC (1992) Sequence discrimination by alternatively spliced isoforms of a DNA binding zinc finger domain. Science 257:1951-1955.

Goldman AL, Van der Goes van Naters W, Lessing D, Warr CG, Carlson JR (2005) Coexpression of two functional odor receptors in one neuron. Neuron 45:661-666.

Hallem EA, Carlson JR (2006) Coding of odors by a receptor repertoire. Cell 125:143-160.

Hsu T, Gogos JA, Kirsh SA, Kafatos FC (1992) Multiple zinc finger forms resulting from developmentally regulated alternative splicing of a transcription factor gene. Science 257:1946-1950.

Ingraham HA, Flynn SE, Voss JW, Albert VR, Kapiloff MS, Wilson L, Rosenfeld MG (1990) The POU-specific domain of Pit-1 is essential for sequencespecific, high affinity DNA binding and DNA-dependent Pit-1-Pit-1 interactions. Cell 61:1021-1033.

Jacobson EM, Li P, Leon-del-Rio A, Rosenfeld MG, Aggarwal AK (1997) Structure of Pit-1 POU domain bound to DNA as a dimer: unexpected arrangement and flexibility. Genes Dev 11:198-212.

Jefferis GS, Vyas RM, Berdnik D, Ramaekers A, Stocker RF, Tanaka NK, Ito K, Luo L (2004) Developmental origin of wiring specificity in the olfactory system of Drosophila. Development 131:117-130.

Jhaveri D, Sen A, Rodrigues V (2000) Mechanisms underlying olfactory neuronal connectivity in Drosophila: the atonal lineage organizes the pe- riphery while sensory neurons and glia pattern the olfactory lobe. Dev Biol 226:73-87.

Kemler I, Schreiber E, Müller MM, Matthias P, Schaffner W (1989) Octamer transcription factors bind to two different sequence motifs of the immunoglobulin heavy chain promoter. EMBO J 8:2001-2008.

Klemm JD, Rould MA, Aurora R, Herr W, Pabo CO (1994) Crystal structure of the Oct-1 POU domain bound to an octamer site: DNA recognition with tethered DNA-binding modules. Cell 77:21-32.

Komiyama T, Johnson WA, Luo L, Jefferis GS (2003) From lineage to wiring specificity. POU domain transcription factors control precise connections of Drosophila olfactory projection neurons. Cell 112:157-167.

Komiyama T, Carlson JR, Luo L (2004) Olfactory receptor neuron axon targeting: intrinsic transcriptional control and hierarchical interactions. Nat Neurosci 7:819-825.

Kreher SA, Kwon JY, Carlson JR (2005) The molecular basis of odor coding in the Drosophila larva. Neuron 46:445-456.

Kreher SA, Mathew D, Kim J, Carlson JR (2008) Translation of sensory input into behavioral output via an olfactory system. Neuron 59:110-124.

Lai SL, Awasaki T, Ito K, Lee T (2008) Clonal analysis of Drosophila antennal lobe neurons: diverse neuronal architectures in the lateral neuroblast lineage. Development 135:2883-2893.

Larsson MC, Domingos AI, Jones WD, Chiappe ME, Amrein H, Vosshall LB (2004) Or83b encodes a broadly expressed odorant receptor essential for Drosophila olfaction. Neuron 43:703-714.

Ray A, van Naters WG, Shiraiwa T, Carlson JR (2007) Mechanisms of odor receptor gene choice in Drosophila. Neuron 53:353-369.

Ray A, van der Goes van Naters W, Carlson JR (2008) A regulatory code for neuron-specific odor receptor expression. PLoS Biol 6:e125.

Sturm RA, Herr W (1988) The POU domain is a bipartite DNA-binding structure. Nature 336:601-604.

Theil T, McLean-Hunter S, Zörnig M, Möröy T (1993) Mouse Brn-3 family of POU transcription factors: a new amino terminal domain is crucial for the oncogenic activity of Brn-3a. Nucleic Acids Res 21:5921-5929.

Theil T, Zechner U, Klett C, Adolph S, Möröy T (1994) Chromosomal localization and sequences of the murine Brn-3 family of developmental control genes. Cytogenet Cell Genet 66:267-271.

Tichy AL, Ray A, Carlson JR (2008) A new Drosophila POU gene, pdm3, acts in odor receptor expression and axon targeting of olfactory neurons. J Neurosci 28:7121-7129.

Turner EE, Jenne KJ, Rosenfeld MG (1994) Brn-3.2: a Brn-3-related transcription factor with distinctive central nervous system expression and regulation by retinoic acid. Neuron 12:205-218.

Verrijzer CP, van Oosterhout JA, van der Vliet PC (1992a) The Oct-1 POU domain mediates interactions between Oct- 1 and other POU proteins. Mol Cell Biol 12:542-551.

Verrijzer CP, Alkema MJ, van Weperen WW, Van Leeuwen HC, Strating MJ, van der Vliet PC (1992b) The DNA binding specificity of the bipartite POU domain and its subdomains. EMBO J 11:4993-5003.

Vigano MA, Staudt LM (1996) Transcriptional activation by Oct-3: evidence for a specific role of the POU-specific domain in mediating functional interaction with Oct-1. Nucleic Acids Res 24:2112-2118.

Voss JW, Wilson L, Rosenfeld MG (1991) POU-domain proteins Pit-1 and Oct-1 interact to form a heteromeric complex and can cooperate to induce expression of the prolactin promoter. Genes Dev 5:1309-1320.

Xiang M, Zhou L, Peng YW, Eddy RL, Shows TB, Nathans J (1993) Brn-3b: a POU domain gene expressed in a subset of retinal ganglion cells. Neuron 11:689-701.

Zhou S, Stone EA, Mackay TF, Anholt RR (2009) Plasticity of the chemoreceptor repertoire in Drosophila melanogaster. PLoS Genet 5:e1000681. 